\title{
COMPATIBLE RELATIONS OF MODULAR AND ORTHOMODULAR LATTICES
}

\author{
DIETMAR SCHWEIGERT
}

\begin{abstract}
Let $L$ be a modular lattice of finite length. $L$ is a projective geometry if and only if $L$ has only trivial tolerances.
\end{abstract}

A binary relation $\theta$ is called a tolerance of an algebra $\mathfrak{A}=(A, \Omega)$ if $\theta$ is reflexive, symmetric and compatible with the operations of $\mathfrak{A}$. The tolerances $D=\{(a, a) \mid a$ $\in A\}$ and $A^{2}$ are called the trivial tolerances of $\mathfrak{A}$. Obviously every congruence relation of $\mathfrak{A}$ is also a tolerance of $\mathfrak{A}$. If $L$ is a lattice then we consider $R=$ $\{(a, b) \mid a, b \in A, a \leqslant b\}$ which is also a compatible relation of $L$. The lattice of the subalgebras $\rho$ with $D \subseteq \rho \subseteq R$ will be denoted by $S$ and the meet operation of $S$ by $\cap$.

These different kinds of relations were already studied by Hashimoto [4] and by Grätzer and E. T. Schmidt, [7], [6]. The following theorem is a generalization of a result of G. Grätzer and E. T. Schmidt concerning congruence relations.

THEOREM 1. Let $T$ be the lattice of tolerances of $L$ and $S$ the lattice of subalgebras $\rho$ with $D \subseteq \rho \subseteq R$. Then $T$ and $S$ are isomorphic.

Proof. We consider the order-preserving function $t: T \rightarrow S$ defined by $t(\eta)=\eta$ $\cap R, \eta \in T$, and furthermore $s: S \rightarrow T$ defined by $s(\rho)=\xi, \rho \in S$, in the following way. $(a, b) \in \xi$ iff $(a \wedge b, b) \in \rho,(a \wedge b, a) \in \rho,(a, a \vee b) \in \rho$ and $(b, a \vee$ $b) \in \rho$. It is clear that $\xi$ is reflexive and symmetric. If $\left(a_{1}, b_{1}\right) \in \xi$ and $\left(a_{2}, b_{2}\right) \in \boldsymbol{\xi}$ then $\left(a_{1} \wedge b_{1}, b_{1}\right) \in \rho$ and $\left(a_{2} \wedge b_{2}, b_{2}\right) \in \rho$ and therefore $\left(\left(a_{1} \wedge b_{1}\right) \vee\left(a_{2} \wedge b_{2}\right)\right.$; $\left.b_{1} \vee b_{2}\right) \in \rho$. As $a_{1} \vee a_{2}>\left(a_{1} \wedge b_{1}\right) \vee\left(a_{2} \wedge b_{2}\right)$ and $b_{1} \vee b_{2} \geqslant\left(a_{1} \wedge b_{1}\right) \vee\left(a_{2}\right.$ $\left.\wedge b_{2}\right)$ we have $\left(\left(a_{1} \vee a_{2}\right) \wedge\left(b_{1} \vee b_{2}\right), b_{1} \vee b_{2}\right) \in \rho$. Similarly we prove the three other conditions and have $\left(a_{1} \vee a_{2}, b_{1} \vee b_{2}\right) \in \xi$. In the same way we can show that $\xi$ is compatible with the operation $\wedge$. The function $s$ is also order-preserving. We have $t \circ s(\rho)=t(\xi)=\xi \cap R$. If $(c, d) \in \xi \cap R$ then we have $(c, d)=(c \wedge$ $d, d) \in \rho$. If $(a, b) \in \rho$ then $(a, b) \in R$ and $(a \wedge b, b) \in \rho,(a \wedge b, a) \in \rho,(a, a \vee$ $b) \in \rho$ and $(b, a \vee b) \in \rho$ and therefore $(a, b) \in \xi \cap R$. We have $t \circ s=1_{s}$ and $s \circ t=1_{T}$ is proved similarly.

THEOREM 2. Let $L$ be an orthomodular lattice. $A$ binary relation $\theta$ of $L$ is $a$ congruence relation if and only if $\theta$ is reflexive, symmetric and compatible with join and meet.

Received by the editors January 21, 1980.

1980 Mathematics Subject Classification. Primary 06C05; Secondary 06C15.

(C) 1981 American Mathematical Society $0002-9939 / 81 / 0000-0126 / \$ 01.75$ 
Proof. As $L$ is relatively complemented $\theta$ is a lattice congruence of $L$ [4], [7]. It remains to show that from $a \theta b$ we have $a^{\prime} \theta b^{\prime}$. We assume $a<b$ and have $a \wedge b^{\prime} \theta b \wedge a^{\prime}$ and hence $b^{\prime} \theta\left(b \wedge a^{\prime}\right) \vee b^{\prime}$. As $b^{\prime}<a^{\prime}$ we get by the orthomodular $b^{\prime} \vee\left(a^{\prime} \wedge b\right)=a^{\prime}$ hence $a^{\prime} \theta b^{\prime}$. If $a \nless b$ then we consider $a \wedge b \theta a \vee b$ and derive $a^{\prime} \wedge b^{\prime} \theta a^{\prime} \vee b^{\prime}$.

REMARK. Every orthomodular lattice $L$ is an algebra of a Malcev variety. If $\theta$ is a tolerance of $L$ and is therefore compatible with the orthocomplementation of $L$ then $\theta$ is also a congruence relation and vice versa [3], [9]. From [8, p. 663, Hilfssatz] we can derive

THEOREM 3. Let $L$ be a relatively complemented ortholattice. $L$ is simple if and only if $L$ has as a lattice only trivial tolerances.

THEOREM 4. If a modular lattice $L$ of finite length has only trivial tolerances then $L$ is atomistic.

Proof. For every element $a \in L$ we define $a^{+}=\inf \{b \mid b \prec a\}$ if $a>0$ and $a^{+}=0$ else. In a modular lattice of finite length we have $(a \vee b)^{+}=a^{+} \vee b^{+}[3$, p. 269, Lemma 6.1(e)]. We consider the following binary relation $\rho=\{(a, b) \mid a<b$, $\left.b^{+} \leqslant a\right\}$ which is reflexive and compatible with join and meet. Obviously we have $D \subseteq \rho \subseteq R$ and as $L$ has only trivial tolerance we conclude from Theorem 1 that $\rho=R$. As $(0,1) \in R$ we have $1^{+}=0$ and hence $L$ is coatomistic and complemented [1, Theorem IV.6].

Theorem 4 and the well-known results on modular geometric lattices give rise to the following theorems.

THEOREM 5. Let $L$ be a modular lattice of finite length. $L$ is a projective geometry if and only if $L$ has only trivial tolerances.

THEOREM 6. Let $L$ be an arguesian lattice of finite length $l, l>3 . L$ is isomorphic to the lattice of all subspaces of a vector space over some division ring if and only if $L$ has only trivial tolerances.

These results cannot be extended to lattices of infinite length. The restriction is necessary since the relation $a \rho b$ iff $a \leqslant b$ and $\operatorname{codim}_{b}(a)<\infty$ will generate a proper nontrivial tolerance relation on the subspace lattice of an infinite-dimensional projective space.

\section{REFERENCES}

1. G. Birkhoff, Lattice theory, 3rd ed., Amer. Math. Soc Colloq. Publ., vol. 25, Amer. Math. Soc., Providence, R. I., 1967.

2. I. Chaida, J. Niederle and B. Zelinka, On existence conditions for compatible tolerances, Czechoslovak Math. J. 26 (1976), 304-311.

3. G. D. Findlay, Reflexive homomorphic relations, Canad. Math. Bull. 3 (1960), 131-132.

4. J. Hashimoto, Congruence relations and congruence classes in lattices, Osaka J. Math. 15 (1963), 71-86.

5. C. Hermann, S-verklebte Summen von Verbänden, Math. Z. 130 (1973), 255-274. 
6. G. Grätzer, General lattice theory, Stuttgart, 1978.

7. G. Grätzer and E. T. Schmidt, On congruence lattices of lattices, Acta Math. Acad. Sci. Hungar. 13 (1962), 178-185.

8. M. Kamara and D. Schweigert, Eine Charakterisierung polynomvollständiger Polaritätsverbände, Arch. Math. (Basel) 30 (1978), 661-664.

9. H. Werner, A Mal'cev condition for admissible relations, Algebra Universalis 3 (1973), 263.

10. R. Wille, Eine Charakterisierung endlicher, ordnungspolynomvollständiger Verbände, Arch. Math. (Basel) 28 (1977), 557-560.

FB Mathematik, Universitüt Kaiserslautern, 675 Kaiserslautern, West Germany 